Classification

Physics Abstracts

81.00

\title{
Sur des propriétés des surfaces de quelques semiconducteurs III-V déduites de mesures de photovoltage
}

\author{
A. Ismail (*), M. T. Chehabeddine (*) et L. Lassabatère \\ Laboratoire d'Etudes des Surfaces, Interfaces et Composants, UA CNRS DO7870, Case \\ Courrier 088, Université de Montpellier II, Place Eugène Bataillon, 34095 Montpellier Cedex 5, \\ France
}

(Reçu le 13 septembre 1991, révisé et accepté le 6 février 1992)

\begin{abstract}
Résumé. - On étudie dans cet article le photovoltage de surface obtenu par éclairement avec des photons d'énergie supérieure au gap du semiconducteur. Après avoir présenté les modèles théoriques, on calcule le photovoltage basé sur ces modèles et détermine, pour quelques cas typiques, l'évolution du photovoltage avec le taux d'injection. On analyse ensuite les résultats expérimentaux que nous avons obtenus par des mesures électriques directes (différence de potentiel de contact) du photovoltage de surface clivée de quelques semiconducteurs III-V. On montre que, moyennant quelques hypothèses simplificatrices raisonnables et des modélisations simples, on peut, à partir des courbes de photovoltage déduire des positions de l'ancrage du niveau de Fermi et des caractéristiques des états de surface.
\end{abstract}

\begin{abstract}
In this paper we study results concerning the surface photovoltage of some cleaved III-V semiconductors illuminated by photon with $h \nu>E_{g}$. We firstly present the theorical models used to determine the photovoltage and then study the variation of this photovoltage with the photon density for typical surface states of III-V semiconductor surfaces. Afterwards experimental results we have obtained by Kelvin measurements are analysed. Using simple hypothesis and model, we show that, from photovoltage curves, information on Fermi level pinning and on the surface state characteristics can be obtained.
\end{abstract}

\section{Introduction.}

L'effet des photons incidents sur la barrière de surface est connu depuis fort longtemps. Il a donné lieu à de nombreuses études théoriques et expérimentales, dont les conclusions, rendues délicates en raison de la complexité des mécanismes pouvant rentrer en jeu et du nombre important de paramètres à prendre en compte, n'ont pas répondu aux espoirs [1-12]. Les expériences basées sur la mesure du photovoltage ont perdu de leur denşité. Le rôle des photons est devenu moins présent à l'esprit et les effets qu'ils pouvaient induire ont été négligés, en particulier dans les études au synchrotron. Des études récentes reconsidérant l'effet des photons (tout comme celui d'électrons incidents) ont montré qu'il fallait

(*) Adresse permanente : Faculté des Sciences I, Université Libanaise, Beyrouth, Liban. 
absolument le prendre en compte pour interpréter les résultats de photoémission [13-14]. De ce fait, les travaux basés sur le photovoltage ont repris un vif intérêt et les résultats qu'ils peuvent fournir sont au centre de recherches récentes sur les surfaces, leurs états, leurs interactions avec gaz et métaux.

Dans cet article, nous exploitons et illustrons l'intérêt que peut présenter la mesure du photovoltage de surface pour l'étude des états de surface et des interfaces en formation. En nous appuyant sur une série de résultats obtenus au laboratoire par éclairement avec des photons d'énergie $h \nu$ supérieure à la bande interdite $E_{\mathrm{g}}$ et concernant quatre semiconducteurs III-V dont l'intérêt en électronique est très important, à savoir GaAs, InP, GaP, GaSb, nous montrons que, moyennant quelques hypothèses simplificatrices raisonnables et des modélisations simples, on peut, à partir des courbes de photovoltage, déduire des caractéristiques des états.

\section{Rappels sur le photovoltage.}

2.1 GÉnÉRALITÉs. - Le photovoltage de surface (SPV) est défini par la différence entre les potentiels de surface du semiconducteur à l'obscurité $V_{\mathrm{s}}$ et sous éclairement $V_{\mathrm{s}}^{*}$. $\mathrm{SPV}=V_{\mathrm{s}}-V_{\mathrm{s}}^{*}$.

Cette modification de la barrière peut être obtenue en éclairant la surface avec des photons d'énergie supérieure à la bande interdite $\left(h \nu>E_{\mathrm{g}}\right)$ ou avec des photons d'énergie inférieure (spectroscopie de photovoltage des états dans la bande interdite).

Ce photovoltage est un paramètre important dans la caractérisation électrique de la surface. $\mathrm{Si}$, son signe et sa valeur, sont souvent utilisés pour fournir des informations sur le sens de la courbure de bande et l'importance de la barrière de surface [15-21] des études plus quantitatives ont été effectuées par plusieurs auteurs qui ont, en particulier, essayé de le formuler en fonction de la barrière. Les relations proposées sont quelquefois différentes à cause de la diversité des modèles de départ et elles ne rendent pas compte de l'ensemble des résultats expérimentaux. Elles montrent cependant qu'en faisant intervenir des paramètres physiques susceptibles d'influencer les mécanismes photovoltaïques (par exemple, densité d'états de surface, section efficace de capture...), des modèles simples peuvent expliquer les résultats de mesures électriques ; on obtient un accord satisfaisant entre courbes expérimentales et théoriques.

2.2 APPROCHE THÉORIQUE. - Nous nous limiterons ici au cas $h \nu>E_{\mathrm{g}}$. Différents mécanismes physiques peuvent intervenir dans la valeur du photovoltage au-dessus du gap. On peut envisager une variation de potentiel de surface $V_{\mathrm{s}}$, soit par modification du nombre de porteurs libres dans la couche superficielle du semiconducteur enrichie en porteurs $(\Delta n=\Delta p)$, accompagnée ou non d'un changement de la charge des états de surface, soit par compensation de la charge des états par migration sous l'effet du champ de charge d'espace des paires électrons-trous créés par l'absorption des photons incidents (dans le cas du type $\mathrm{n}$, $\Delta p>\Delta n$ en surface).

2.2.1 Cas où $\Delta n(x)=\Delta p(x)$ : la charge des états de surface reste inchangée sous éclairement.

- Dans ce cas :

$$
Q_{\mathrm{sc}}^{*}\left(V_{\mathrm{s}}^{*}\right)=Q_{\mathrm{sc}}\left(V_{\mathrm{s}}\right)
$$

$Q_{\mathrm{sc}}\left(V_{\mathrm{s}}\right)$ est la charge totale par unité de surface dans le semiconducteur à l'obscurité. $Q_{\mathrm{sc}}^{*}\left(V_{\mathrm{s}}^{*}\right)$ est la charge totale par unité de surface dans le semiconducteur sous éclairement. 
L'expression détaillée de l'équation (1), déduite de la littérature [1], peut s'écrire sous la forme :

$$
\begin{gathered}
F^{2}\left(v_{\mathrm{s}}^{*}\right)+\frac{4 \Delta n}{n_{\mathrm{b}}+P_{\mathrm{b}}} \operatorname{Ch}\left(v_{\mathrm{s}}^{*}-1\right)=F^{2}\left(v_{\mathrm{s}}\right) \\
v_{\mathrm{s}}=\frac{q V_{\mathrm{s}}}{K T} .
\end{gathered}
$$

avec

L'expression de $F$ est complexe sauf en régime d'appauvrissement et de faible inversion où elle se simplifie et devient:

$$
F_{s}=2\left[\varepsilon\left(v_{s}-1\right)\right]^{1 / 2}
$$

On peut remarquer que seules les valeurs de $v_{\mathrm{s}}^{*}$ telles que $v_{\mathrm{s}}^{*}<v_{\mathrm{s}}$ peuvent satisfaire l'équation (2). Ceci montre que $v_{\mathrm{s}}$ diminue sous éclairement.

En choisissant les valeurs de $v_{\mathrm{s}}$ et le niveau d'injection $\frac{\Delta n}{n_{\mathrm{b}}}$ l'équation (2) résolue numériquement fournit la valeur de $V_{\mathrm{s}}^{*}$ et donc la valeur du photovoltage $V_{\mathrm{s}}-V_{\mathrm{s}}^{*}$. Cette possibilité est illustrée figures 1 et 2 .

Le semiconducteur utilisé dans cet exemple est le GaAs de type $n$, de dopage $5 \times 10^{16} \mathrm{~cm}^{-3}$ Le photovoltage est représenté, pour des valeurs de $V_{\mathrm{s}}$ données, en fonction du taux d'injection des porteurs excédentaires $\frac{\Delta n}{n_{\mathrm{b}}}$, figure 1 . Il est d'autant plus important que $V_{\mathrm{s}}$ et $\frac{\Delta n}{n_{\mathrm{b}}}$ sont élevés; et sa croissance est moins rapide pour $V_{\mathrm{s}}$ faible.

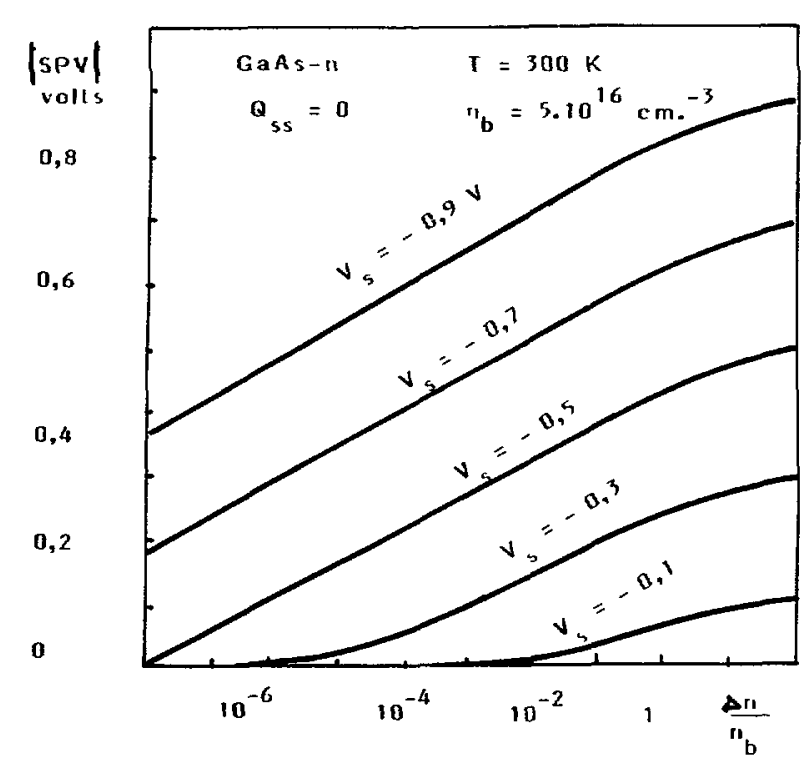

Fig. 1. - Variation du photovoltage de surface en fonction du taux d'injection $\Delta n / n_{\mathrm{b}}$ avec $V_{\mathrm{s}}$ comme paramètre.

[Surface photovoltage variations versus $\Delta n / n_{b}$ for different barrier heights.] 


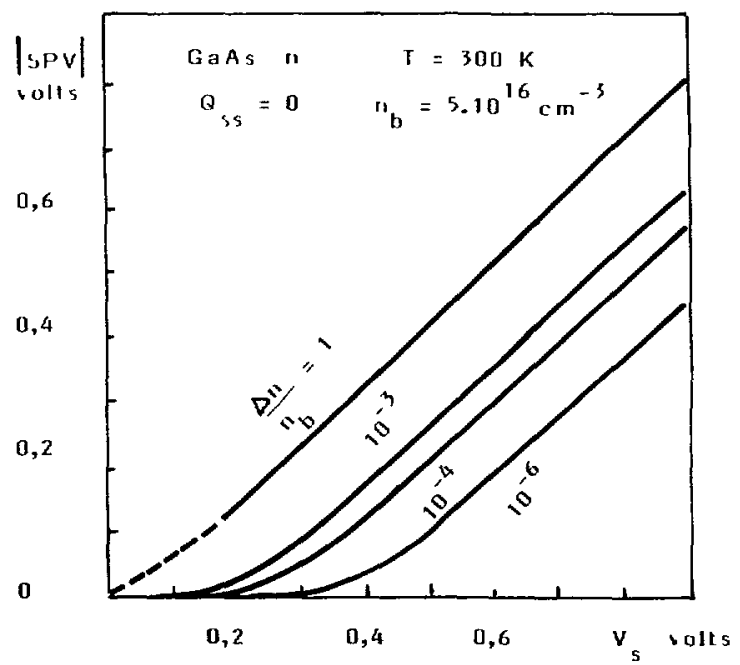

Fig. 2. - Variation du photovoltage de surface en fonction de la barrière $V_{\mathrm{s}}$ avec $\Delta n / n_{\mathrm{b}}$ comme paramètre.

[Surface photovoltage variations versus $V_{\mathrm{s}}$ for different values of $\Delta n / n_{\mathrm{b}}$.]

La figure 2 montre de plus que dans la gamme des taux d'injection $\frac{\Delta n}{n_{\mathrm{b}}}$ utilisés expérimentalement, on peut s'attendre à ce qu'il n'y ait pas de photovoltage dans le cas de faibles barrières de surface $(0,1$ à $0,2 \mathrm{~V})$. Cette évolution de $\mathrm{SPV}$ en fonction de $V_{\mathrm{s}}$ et $\frac{\Delta n}{n_{\mathrm{b}}}$ s'explique facilement si l'on compare la densité d'électrons en surface sous éclairement $n_{\mathrm{s}}^{*}$ et dans l'obscurité. Le calcul montre que le photovoltage ne devient sensible que si $\Delta n \geqslant n_{\mathrm{s}}$ soit $\left(\frac{\Delta n}{n_{\mathrm{b}}} \geqslant \mathrm{e}^{v_{\mathrm{s}}}\right)$. Dans le cas d'une déplétion, au fur et à mesure que la barrière de surface diminue soús éclairement, l'injection nécessaire pour se rapprocher des bandes plates, devient plus importante. Enfin le photovoltage dépend du dopage du semiconducteur comme le montre la figure 3. Pour une même valeur de $\Delta n$, il diminue lorsque le dopage augmente.

Dans la gamme des taux d'injection utilisés expérimentalement, les valeurs du photovoltage obtenues par ce modèle, bien que fréquemment en accord avec des valeurs expérimentales, ne rendent cependant pas compte de l'ensemble des valeurs observées. Aussi ce modèle ne donne pas entièrement satisfaction et il faut fréquemment prendre en compte des modifications de la charge de surface sous éclairement.

2.2.2 Cas où $\Delta n(x)=\Delta p(x)$ : la charge des états de surface est modifiée sous éclairement. Nous considérons, dans le calcul suivant, le cas où l'occupation des états est modifiée sous éclairement par échange des charges entre les états et les bandes. La charge dans les états est alors déterminée en utilisant la fonction d'occupation hors équilibre $f_{t}^{*}$ donnée par l'expression :

$$
f_{\mathrm{t}}^{*}=\frac{c_{\mathrm{n}} n_{\mathrm{s}}+p_{1} c_{\mathrm{p}}}{c_{\mathrm{n}}\left(n^{*}+n_{1}\right)+c_{\mathrm{p}}\left(p_{\mathrm{s}}+p_{1}^{*}\right)}
$$




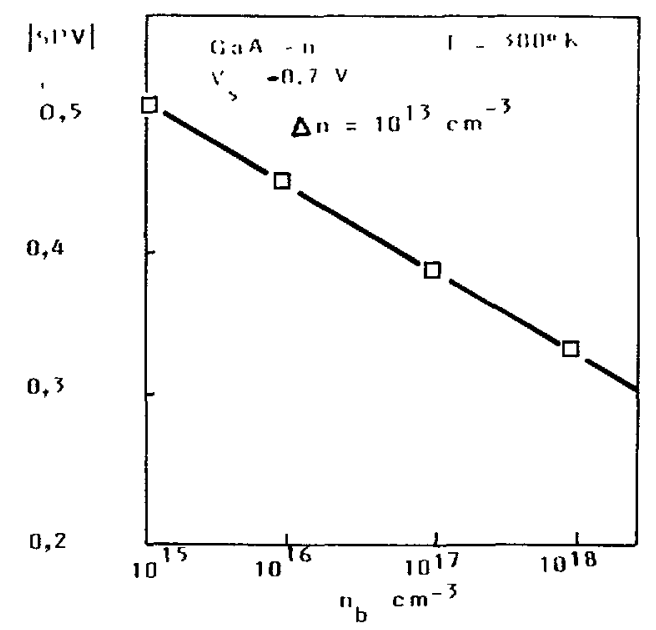

Fig. 3. - Variation du photovoltage en fonction du dopage du semiconducteur.

[Photovoltage variations versus semiconductor doping.]

$c_{\mathrm{n}}$ et $c_{\mathrm{p}}$ sont respectivement les probabilités par unité de temps pour qu'un électron et un trou soient capturés par un état de surface.

$n_{1}$ et $p_{1}$ sont respectivement les densités des électrons et des trous lorsque le niveau de Fermi coïncide avec le niveau de l'état $E_{\mathrm{t}}$.

La variation de la charge des états dans le cas des différents types d'indice $i$, s'écrit donc $q \sum N_{\mathrm{t}}^{(i)}\left(f_{\mathrm{t}}-f_{\mathrm{t}}^{*}\right)^{(t)}$ où $f_{\mathrm{t}}$ est la fonction d'occupation à l'équilibre et $N_{\mathrm{t}}$ est la densité d'état.

L'équation (1) par laquelle on détermine $V_{\mathrm{s}}^{*}$ devient dans le cas simple de deux états discrets (état donneur et état accepteur) :

$$
Q_{\mathrm{sc}}^{*}\left(V_{\mathrm{s}}^{*}\right)+q N_{\mathrm{d}}\left(f_{\mathrm{t}}-f_{\mathrm{t}}^{*}\right)+q N_{\mathrm{a}}\left(f_{\mathrm{t}}-f_{\mathrm{t}}^{*}\right)=Q_{\mathrm{sc}}\left(V_{\mathrm{s}}\right) .
$$

Cette équation qui fait intervenir la position énergétique des états, leur densité, leur section efficace de capture, peut être résolue numériquement.

Les figures 4 et 5 relatives à une surface de GaAs avec respectivement un et deux états illustrent les résultats qu'on peut déduire de la relation 5 .

Les courbes sont paramétrées en fonction du rapport $\frac{c_{\mathrm{p}}}{c_{\mathrm{n}}}$ Les valeurs de $\frac{c_{\mathrm{p}}}{c_{\mathrm{n}}}$ utilisées correspondent à des ordres de grandeurs couramment admis pour les sections efficaces de capture des centres attractifs, répulsifs ou neutres [1]. Elles sont de 1'ordre de $10^{-10}$ pour un accepteur et $10^{-3}$ pour un donneur. Elles doivent donc être considérées comme indicatives car malheureusement on ne connaît pas les valeurs réelles de $c_{\mathrm{n}}$ et $c_{\mathrm{p}}$.

La figure 4 correspond au cas où un seul état accepteur est présent en surface. On note que dans le cas où $c_{\mathrm{p}} \ll c_{\mathrm{n}}$, le photovoltage peut être notablement réduit. Sa valeur tombe dans la fourchette des valeurs que nous avons observées sur GaAs dans la gamme des taux d'injection utilisés (zone hachurée).

La figure 5 relative à deux états montre que, pour les valeurs de $\frac{c_{\mathrm{p}}}{c_{\mathrm{n}}}$ envisagées précédemment, le photovoltage reste du même ordre de grandeur que dans le cas où $Q_{\text {ss }}$ est constante dans la gamme des taux d'injection utilisés expérimentalement. De façon 


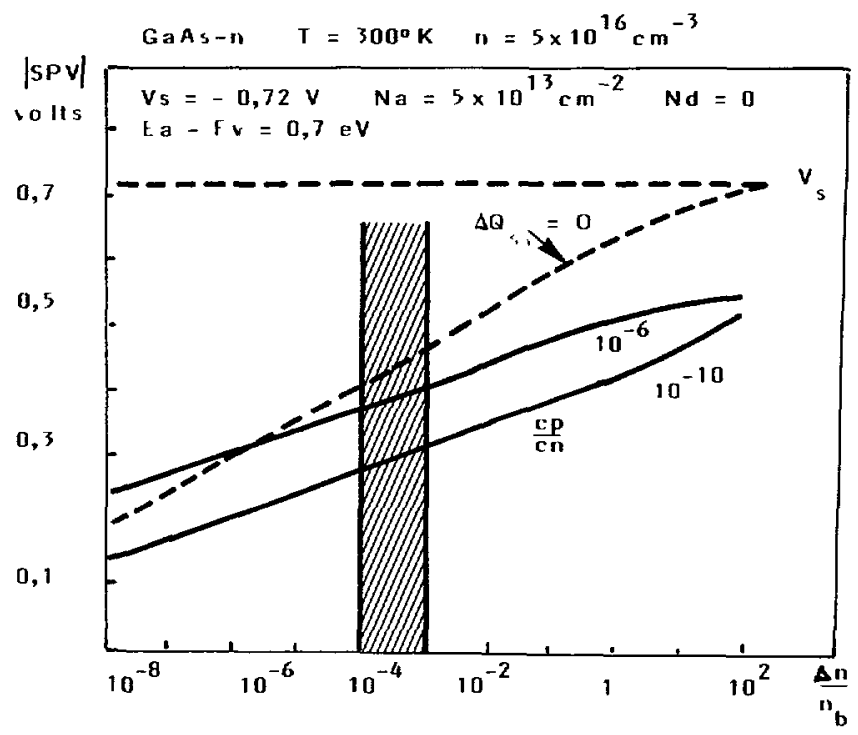

Fig. 4. - Evolution du photovoltage en fonction du taux d'injection $\Delta n / n_{\mathrm{b}}$ dans le cas où $\Delta Q_{s s} \neq 0$ avec un état accepteur et $C_{p} / C_{n}$ de l'accepteur comme paramètre.

[Surface photovoltage variations versus $\Delta n / n_{\mathrm{b}}$ for $\Delta Q_{\mathrm{ss}} \neq 0$. One acceptor state is present at the surface and its $C_{\mathrm{p}} / C_{\mathrm{n}}$ value is taken as parameter.]

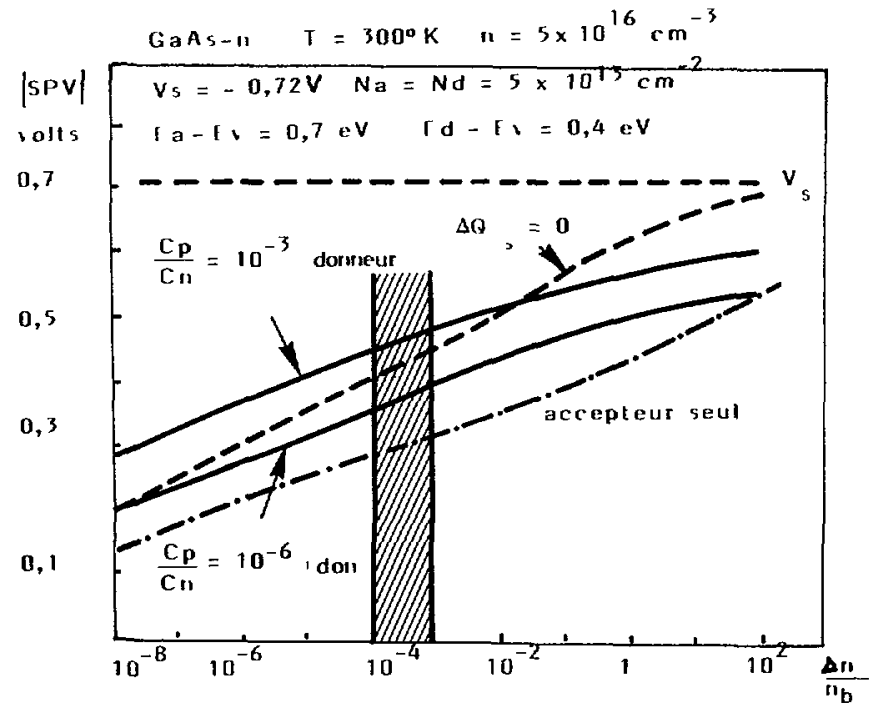

Fig. 5. - Evolution du photovoltage en fonction du taux d'injection $\Delta n / n_{\mathrm{b}}$ dans le cas où $\Delta Q_{\mathrm{ss}} \neq 0$ avec deux états : un accepteur et un donneur : $C_{\mathrm{p}} / C_{\mathrm{n}}$ de l'accepteur est fixé : $C_{\mathrm{p}} / C_{\mathrm{n}}$ du donneur est un paramètre. $C_{\mathrm{p}} / C_{\mathrm{a}}$ (accepteur) $=10^{-10}$

[Photovoltage variations versus the injection ratio $\Delta n / n_{\mathrm{b}}$ for $\Delta Q_{s \mathrm{~s}}=0$. One acceptor state and one donor state are simultaneously present at the surface. $C_{n} / C_{p}$ of the acceptor is fixed $\left(10^{-10}\right) . C_{p} / C_{n}$ of the donor is the parameter.] 
générale, on note que le photovoltage est d'autant plus faible que $c_{\mathrm{p}} \ll c_{n}$, aussi bien pour les accepteurs que pour les donneurs. Dans tous les cas, le photovoltage croît moins rapidement avec le taux d'injection lorsque la charge des états varie. De ce fait, les taux d'injection nécessaires pour atteindre les conditions des bandes plates sont trop élevés pour être obtenus avec des sources classiques de lumière. On ne peut donc atteindre directement la hauteur totale de barrière.

La comparaison des courbes précédentes avec celles obtenues expérimentalement montre cependant que les variations expérimentales du photovoltage (Figs. 10 et 11), dans le cas de surfaces clivées avec états intrinsèques ou extrinsèques peuvent être analysés avec ce modèle.

2.2.3 Cas où $\Delta n(x) \neq \Delta p(x)$. - Ce cas correspond à la présence d'un gradient de porteurs excédentaires dans le semiconducteur correspondant à l'accumulation des minoritaires en surface et/ou à proximité de la surface. Le mécanisme envisagé est schématisé dans la figure 6 .

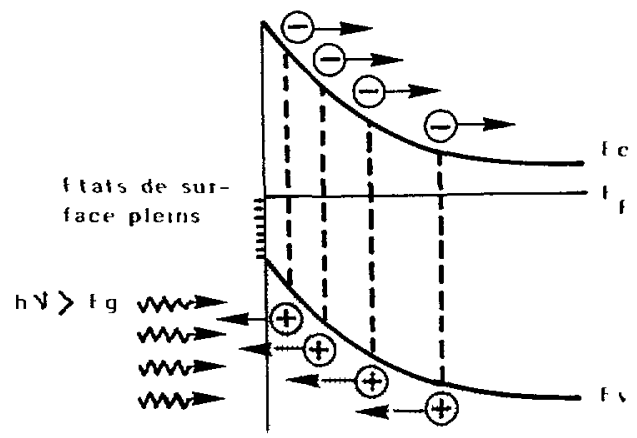

Fig. 6. - Mécanisme envisagé du photovoltage dans le cas où $n(x) \neq p(x)$.

[Surface photovoltage mechanism envisaged for $n(x)=p(x)$.]

Les charges excédentaires générées par les photons incidents, au voisinage de la surface, sont soumises au champ de la charge d'espace qui entraine les minoritaires vers la surface et les majoritaires vers le volume.

Au fur et à mesure que la charge transférée augmente, le champ qui sépare et entraîne les paires électrons-trous s'affaiblit et le photovoltage tend vers une saturation. Le calcul de l'abaissement de la barrière tenant compte de ce mécanisme est complexe.

Une façon d'aborder le problème consiste, comme l'ont fait Spicer et al. [8], à considérer que le photovoltage mesuré n'est autre que la différence de potentiel à circuit ouvert $V_{\text {co }}$ aux bornes d'une diode Schottky sous éclairement.

L'expression de $V_{\text {co }}$ peut être tirée de la relation courant-tension d'une diode Schottky idéale sous éclairement d'intensité $I$ et en circuit ouvert (en négligeant le courant des majoritaires excédentaires et en supposant un rendement quantique égal à 1) :

$$
A^{*} T^{2} \exp \frac{-\phi_{\mathrm{B}}}{K T}\left(\exp \frac{q V_{\mathrm{co}}}{K T}-1\right)-q I=0 .
$$

Soit en négligeant 1 devant le terme en exponentielle :

$$
V_{\mathrm{co}_{\max }}=\frac{\phi_{\mathrm{B}}}{q}-\frac{K T}{q} \ln \frac{A^{*} T^{2}}{q I}
$$


Dans cette relation $V_{\text {co }}$ varie logarithmiquement avec $I$ et dépend des propriétés du substrat à travers $A^{*}$ seulement. Sa variation en fonction du flux de photons incidents pour une barrière initiale de $0,8 \mathrm{eV}$ dans GaAs-n est donnée figure 7 . On voit que sa valeur pour des flux de photons compris entre $5 \times 10^{16}$ et $5 \times 10^{17}$ photons $\mathrm{cm}^{-2} \mathrm{~s}^{-1}$ est comprise entre 300 et $370 \mathrm{mV}$. Ces résultats ne rendent pas compte de toutes les valeurs expérimentales obtenues.

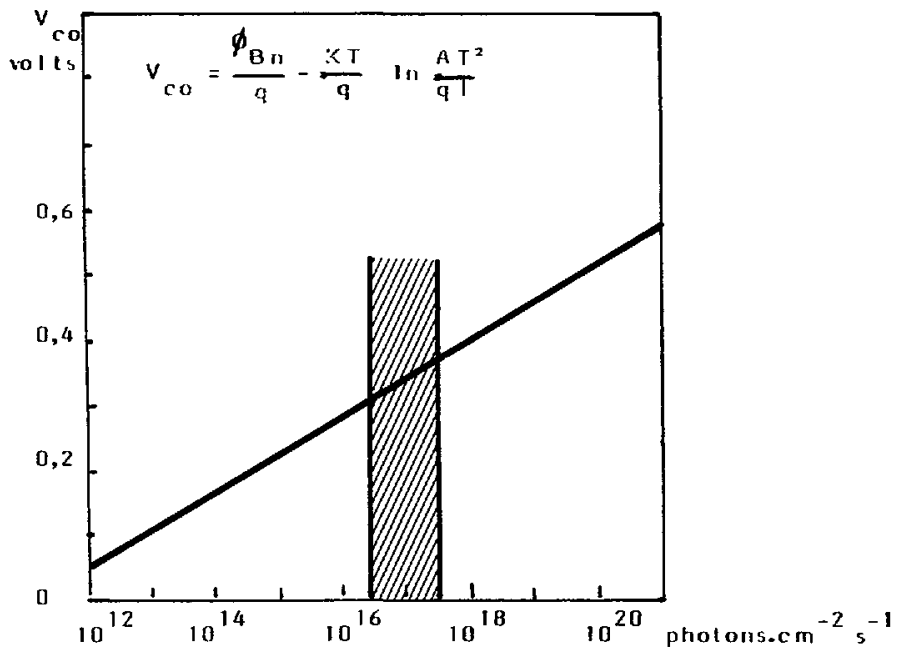

Fig. 7. - Variation de $V_{\text {co }}$ en fonction du flux de photons incidents pour une barrière de $0,8 \mathrm{eV}$ dans GaAs-n.

[ $V_{\text {co }}$ variations versus the photons flux for $V_{\mathrm{s}}=0.8 \mathrm{eV}$. Semiconductor: GaAs $\mathrm{n}$ type.]

\section{Etudes expérimentales et discussion.}

Les résultats que nous présentons sont relatifs à des semiconducteurs III-V : GaAs, InP, $\mathrm{GaSb}$ et $\mathrm{GaP}$ dont les dopages à $300 \mathrm{~K}$ sont donnés dans le tableau $\mathrm{I}$.

Tableau I. - Type et dopage des échantillons.

[Type and doping of the samples.]

\begin{tabular}{|c|c|c|c|c|}
\cline { 2 - 5 } \multicolumn{1}{c|}{} & GaAs & InP & GaSb & GaP \\
\hline Type n $\left(\mathrm{cm}^{-3}\right)$ & $5 \times 10^{15}$ & $7,5 \times 10^{16}$ & $10^{18}$ & $5 \times 10^{15}$ \\
\hline Type p $\left(\mathrm{cm}^{-3}\right)$ & $2,5 \times 10^{15}$ & $5,7 \times 10^{16}$ & $7,6 \times 10^{16}$ & - \\
\hline
\end{tabular}

Ces échantillons qui se présentent sous forme des barreaux de section $8 \times 4 \mathrm{~mm}^{2}$ sont clivés sous ultra-vide $\left(10^{-10}\right.$ Torr ) et caractérisés par des études topographiques du travail de sortie (toutes sources d'ions dans 1'enceinte d'ultravide étaient éteintes). 
La mesure du photovoltage est effectuée par la méthode du condensateur vibrant (sonde Kelvin) qui permet la mesure de la différence de potentiel de contact entre le semiconducteur et l'électrode de référence, c'est-à-dire la mesure du travail de sortie du semiconducteur $\phi_{\text {sc }}$ par rapport à l'électrode de référence. Cette électrode, une boule d'or de diamètre de $1 \mathrm{~mm}$, peut être déplacée le long de la surface à étudier, ce qui permet d'effectuer des topographies du travail de sortie avec une résolution spéciale de $300 \mu \mathrm{m}$ environ et une sensibilité de $1 \mathrm{mV}(22)$.

Une lampe de $100 \mathrm{~W}$ à filament de tungstène permet d'envoyer sur l'échantillon des photons d'énergie plus grande que le gap et de flux de $1^{1}$ ordre de $5 \times 10^{17}$ photons $\mathrm{cm}^{-2} \mathrm{~s}^{-1}$. Un dispositif électronique monté en chaîne de régulation permet l'asservissement de l'intensité lumineuse ou du nombre de photons. On s'affranchit ainsi des difficultés d'interprétation liées au propre spectre de la lampe.

3.1 SuRfACES VIERGES. - Les résultats des mesures du travail de sortie et du photovoltage des surfaces de quatre semiconducteurs effectuées juste après clivage sont reproductibles et se résument comme suit :

- La variation du travail de sortie $\varphi\left(\phi_{n}\right.$ pour type $n, \phi_{p}$ pour type $\left.p\right)$ est étroitement liée aux défauts de clivage et en particulier à la présence de marches comme le montre l'étude en microscopie électronique à balayage. Ces défauts tendent à rapprocher $\phi_{\mathrm{n}}$ et $\phi_{\mathrm{p}}$ (mais $\phi_{\mathrm{n}}$ reste toujours inférieur à $\phi_{\mathrm{p}}$ ). Sur le même type du semiconducteur, les zones présentant une densité de défauts de clivage élevés correspondent aux valeurs de $\phi$ les plus grandes s'il s'agit d'un type $n$ et $\phi$ les plus faibles s'il s'agit d'un type p. Les topographies de $\phi$ sur les clivages ayant des aspects voisins (même qualité de clivage) sont plus uniformes sur type $p$ que sur type $n$ dans le cas de GaAs et GaSb et sur type $n$ que sur type $p$ dans le cas d'InP.

Le photovoltage de surface SPV est positif ou nul sur type $p$, et augmente quand $\phi_{\mathrm{p}}$ diminue. Sur type $n$, il est négatif ou nul et augmente en valeur absolue quand $\phi_{\mathrm{n}}$ augmente. Le photovoltage est dans tous les cas réversible, c'est-à-dire que dès que le flux des photons est arrêté, le travail de sortie reprend sa valeur initiale.

Le signe du photovoltage indique que les types $n$ et $p$ sont en déplétion. Des exemples de variation de $\phi_{\mathrm{n}}$ et $\phi_{\mathrm{p}}$ sous éclairement sont donnés figures 8 et 9 . L'étude de la variation du photovoltage, pour un point donné, en fonction du nombre de photons incidents $\left(h \nu>E_{\mathrm{g}}\right)$ montre que le SPV tend vers la saturation pour un éclairement de $5 \times$ $10^{17}$ photons $\mathrm{cm}^{-2} \mathrm{~s}^{-1}$ (Figs. 10 et 11 ), ce qui correspond aux conditions d'éclairement dans nos mesures de SPV.

- Ces valeurs du photovoltage sont en étroite corrélation avec celles du travail de sortie comme le montrent les courbes représentées figures $12,13,14$ et 15 , courbes qui donnent les variations de SPV avec $\phi$. C'est donc la barrière de surface qui est essentiellement responsable des variations de $\phi$ sur les surfaces clivées propres des semiconducteurs III-V étudiés. La contribution de l'affinité électronique à ces variations de $\phi$ est généralement négligeable. En effet les irrégularités du travail de sortie, associées aux défauts de clivage, ainsi que le photovoltage, étant importants sur GaSb et GaAs de type $n$, InP de type $p$ et faibles sur GaSb, GaAs de type $p$, InP de type $n$, on peut en conclure que, suivant le type, des défauts similaires induisent ou $n$ 'induisent pas des variations de barrière et de travail de sortie. Le fait que pour l'un des types les topographies soient pratiquement plates et le photovoltage faible, permet de conclure que les modifications d'affinité électronique sont négligeables. Numériquement, la dispersion des points de mesure qu'on peut attribuer aux variations de $\chi$ ne dépasse pas quelques dizaines de millivolts, ce qui est très inférieur aux hauteurs de barrière. On peut donc les négliger sans commettre une erreur importante. 


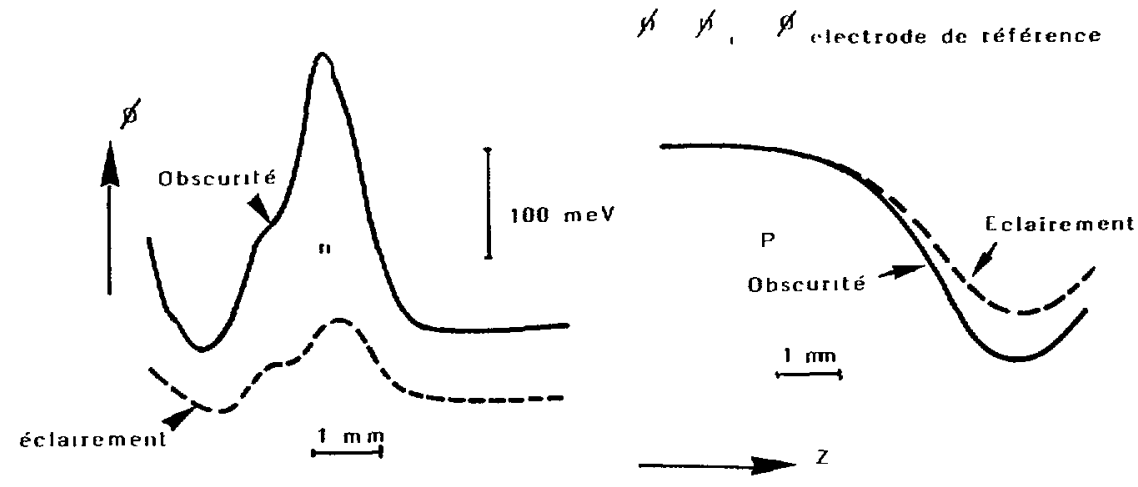

Fig. 8. - Exemples de topographie du travail de sortie de surface de GaAs (110) clivée à l'obscurité et sous éclairement.

[Examples of GaAs workfunction topographies in the dark and under light.]

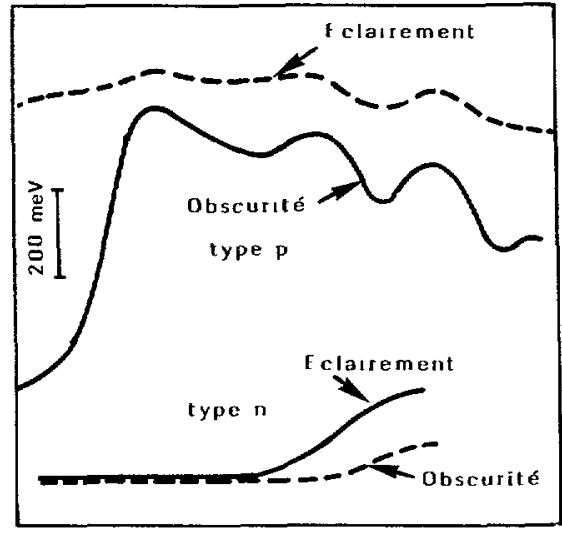

Fig. 9. - Variation du travail de sortie sous l'effet de la lumière de deux surfaces clivées d'InP type n et type p.

[Workfunction variations induced by light for $\mathrm{n}$ and $\mathrm{p}$ type InP cleaved surfaces.]

Dans ces conditions et en désignant pour $\phi_{n}$ le travail de sortie du semiconducteur, en un point quelconque de la surface, $\phi_{\text {on }}$, sa valeur à bandes plates, on a :

$$
\phi_{\mathrm{n}}=\chi+\left(E_{\mathrm{c}}-E_{\mathrm{F}}\right)-q V_{\mathrm{s}}=\phi_{\mathrm{on}}-q V_{\mathrm{s}} .
$$

$\phi_{\mathrm{n}}$ tend vers $\phi_{\text {on }}$ quand $V_{\mathrm{s}}$ tend vers zéro.

On peut donc par extrapolation, à partir des courbes de photovoltage, figure (13), déduire $\phi_{\text {on }}$ (ou $\phi_{\text {op }}$ pour type p) ou plus exactement $\phi_{\text {osc }}-\phi_{\text {électrode }}=\phi_{\text {osc }}-\phi_{\mathrm{m}}$.

Partant d'un échantillon de type $\mathrm{p}$ et d'un échantillon de type $\mathrm{n}$, étudiés avec la même électrode, on pourra donc atteindre :

$$
\left(\phi_{\mathrm{op}}-\phi_{\mathrm{m}}\right)-\left(\phi_{\mathrm{on}}-\phi_{\mathrm{m}}\right)=\phi_{\mathrm{op}}-\phi_{\mathrm{on}} .
$$




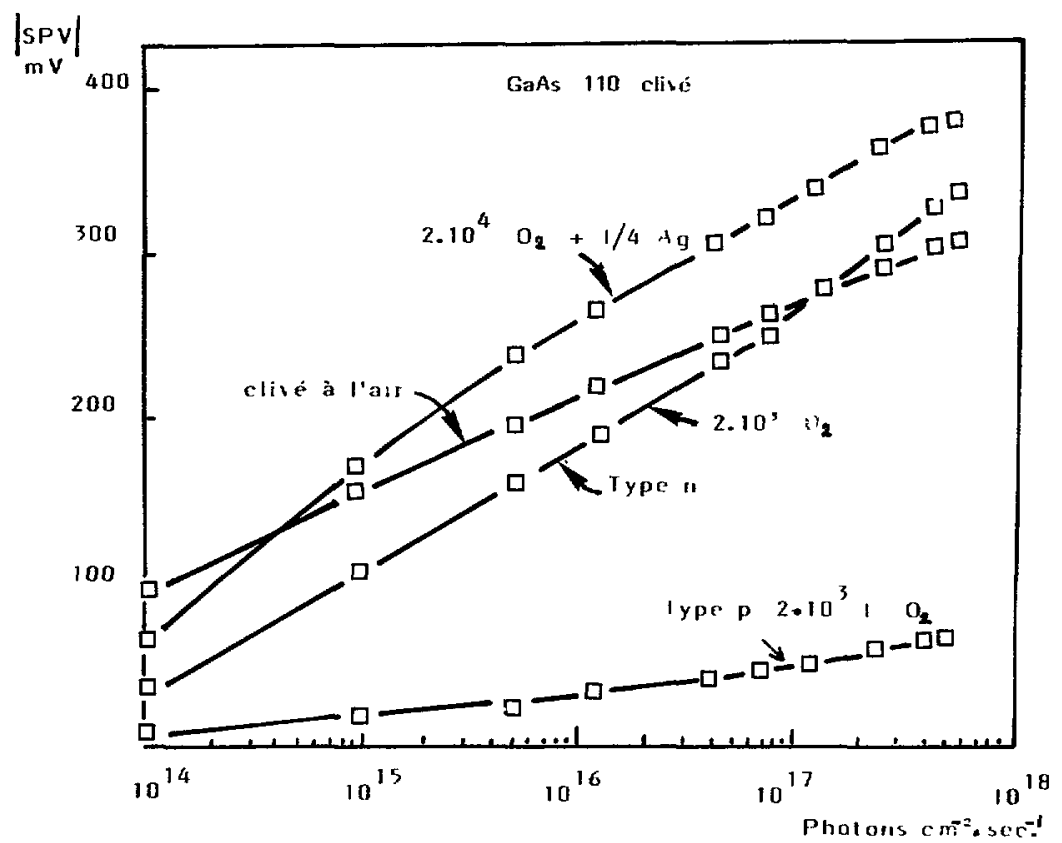

Fig. 10. - Variation du photovoltage en fonction du nombre de photons incidents. Cas de GaAs clivé. [Photovoltage variations versus the photon flux for cleaved GaAs.]

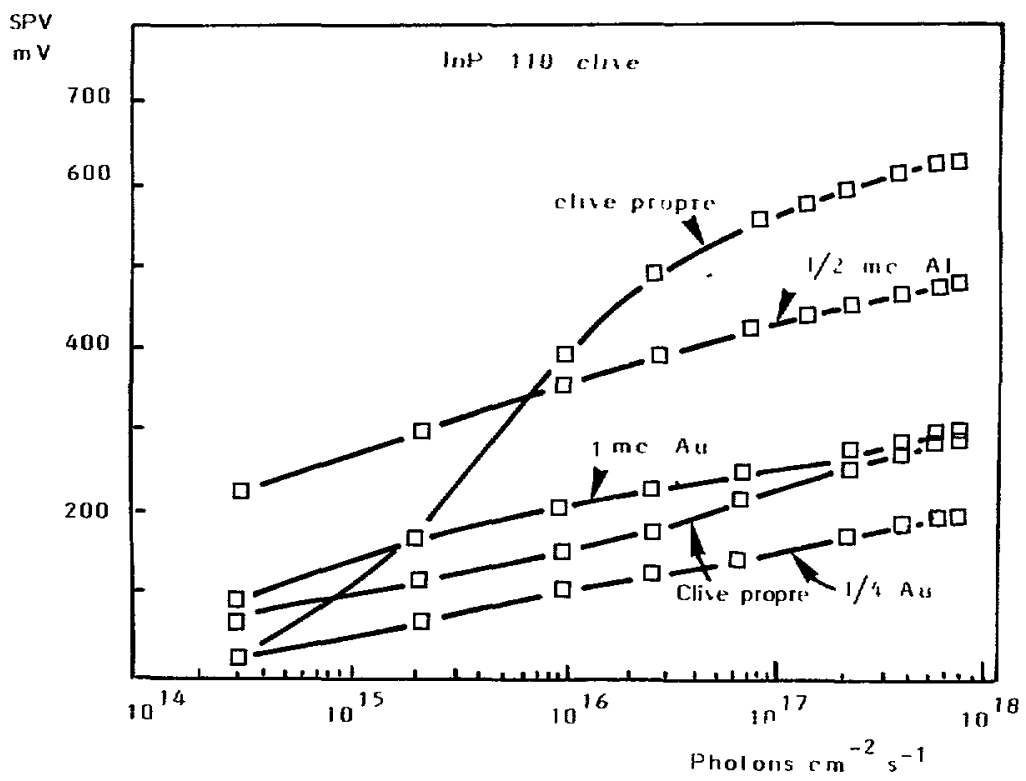

Fig. 11. - Variation du photovoltage en fonction du nombre de photons incidents. Cas d'InP clivé. [Photovoltage variations versus the photon flux for cleaved InP.] 

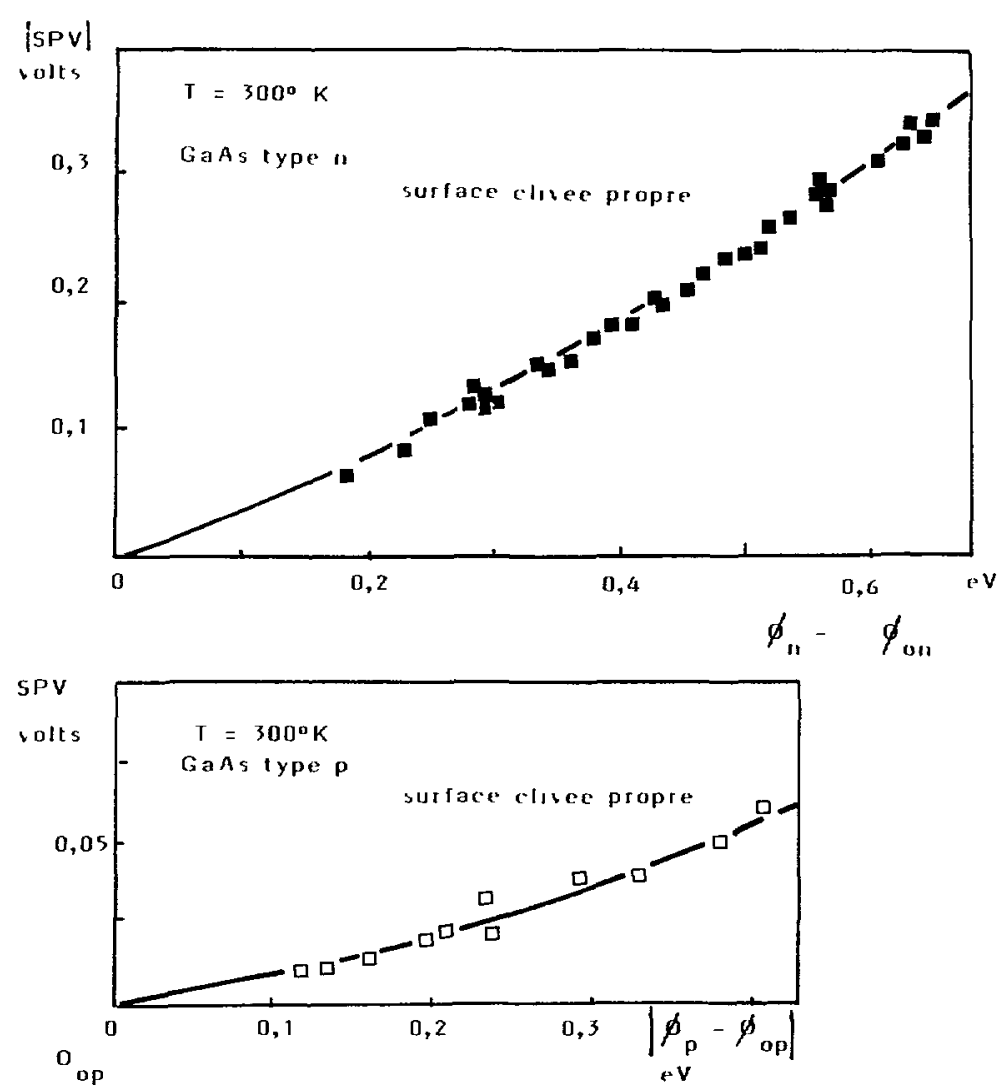

Fig. 12. - Exemples de variations du photovoltage en fonction du travail de sortie mesuré en prenant comme origine le travail de sortie du semiconducteur en bandes plates.

[Variations of the photovoltage versus the workfunction for $\mathrm{n}$ and $\mathrm{p}$ type GaAs.]
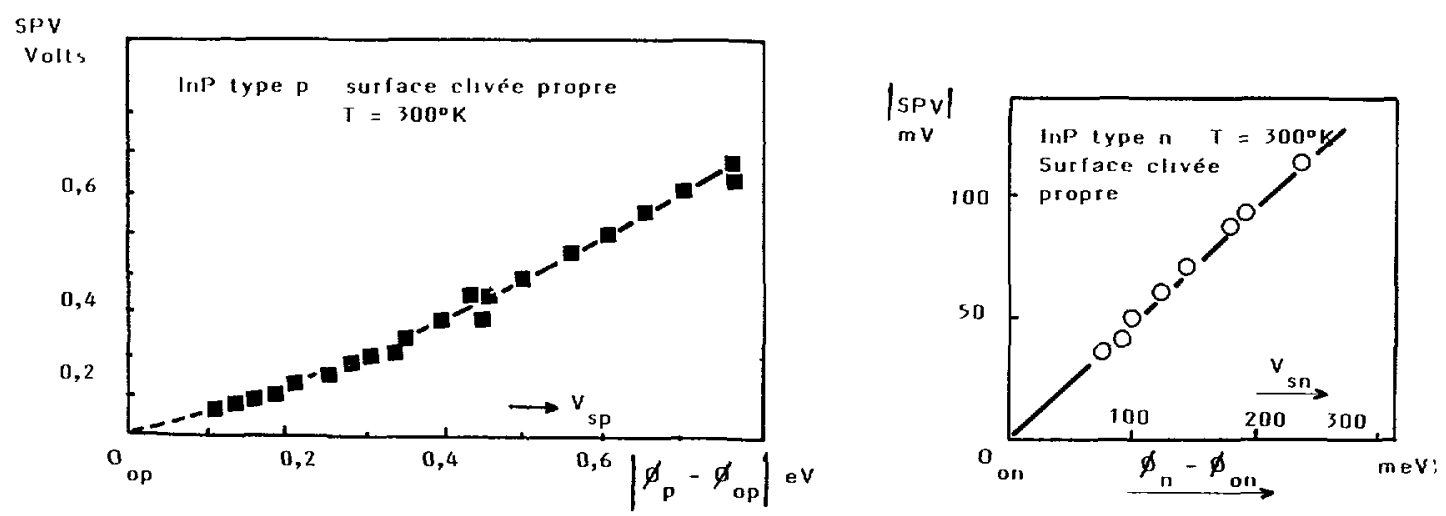

Fig. 13. - Variation du photovoltage d'InP de type $\mathrm{p}$ et $\mathbf{n}$ en fonction du travail de sortie en prenant comme origine le travail de sortie en bandes plates $\phi_{\text {op }}$ pour le type $p$ et $\phi_{\text {on }}$ pour le type $n$.

[Variation of the photovoltage versus the workfunction for $\mathrm{n}$ and $\mathrm{p}$ type InP.] 


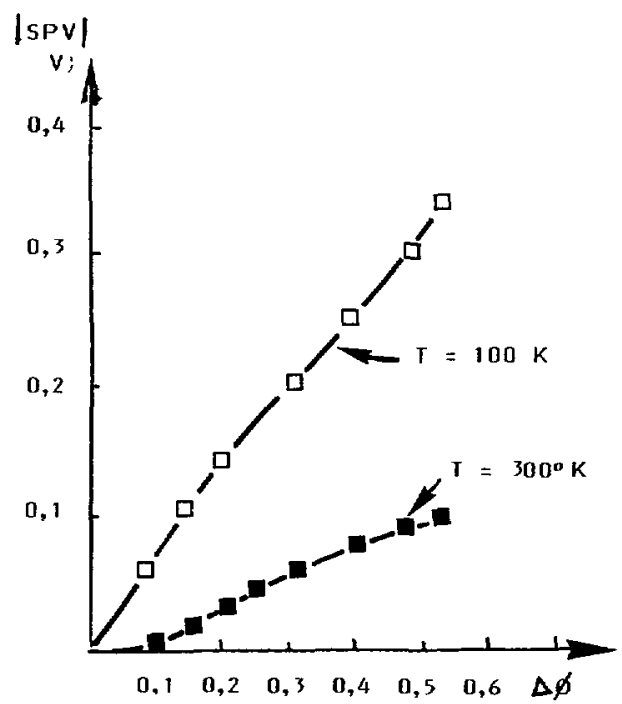

Fig. 14. - Variation du photovoltage en fonction du travail de sortie $\Delta \phi$ (par rapport à l'électrode de référence). Mesures effectuées à $100 \mathrm{~K}$ et $300 \mathrm{~K}$.

[Photovoltage variations versus workfunction variations for $T=100 \mathrm{~K}$ and $T=300 \mathrm{~K}$.]

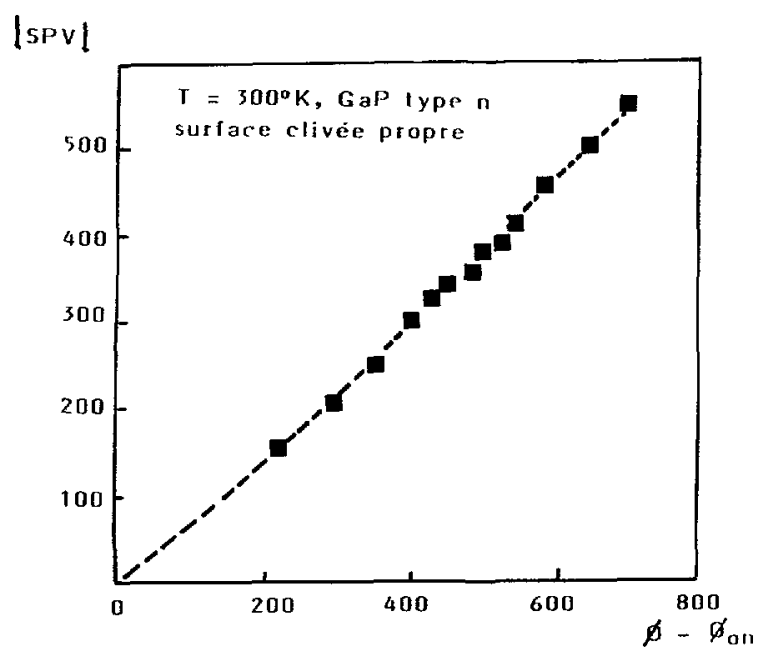

Fig. 15. - Variation du photovoltage en fonction du travail de sortie en prenant comme origine le travail de sortie en bandes plates $\phi_{\text {on }}$.

[Photovoltage variations versus the workfunction for $\mathrm{n}$ type GaP.]

Cette quantité représente précisément la différence entre les niveaux de Fermi des deux échantillons :

$$
\phi_{\mathrm{op}}-\phi_{\mathrm{on}}=\left\lfloor E_{\mathrm{Fn}}-E_{\mathrm{Fp}}\right\rfloor \text {. }
$$

Les valeurs déduites des courbes expérimentales sont très voisines des valeurs caractéristiques des propriétés volumiques des échantillons : on peut donc dire que la méthodologie est 
cohérente et de ce fait détermine directement, à partir de la valeur de $\phi$ en un point quelconque de la surface, la hauteur de barrière $V_{\mathrm{s}}$, et à partir de la différence $\phi_{\mathrm{p}}-\phi_{\mathrm{n}}$, la quantité $V_{\mathrm{sp}}-V_{\mathrm{sn}}=\left(\phi_{\mathrm{op}}-\phi_{\mathrm{on}}\right)-\left(\phi_{\mathrm{p}}-\phi_{\mathrm{n}}\right)$.

Des valeurs numériques de ces quantités mesurées dans quelques cas expérimentaux types, sont données tableaux II, III, IV et V.

Tableau II. - GaAs : $\phi_{\mathrm{n}}-\phi_{\mathrm{on}}=$ Différence entre le travail de sortie $\phi_{\mathrm{n}}$ et le travail de sortie en bandes plates $\phi_{\mathrm{on}} . V_{\mathrm{sp}}-V_{\mathrm{sn}}=$ Somme des barrières sur type $\mathrm{n}$ et $\mathrm{p} . \mathrm{SPV}_{\mathrm{n}}, \mathrm{SPV}_{\mathrm{p}}=$ Photovoltage de surface sur type $\mathrm{n}$ et $\mathrm{p}$.

[GaAs: $\phi_{\mathrm{n}}-\phi_{\mathrm{on}}=$ Difference between the work function $\phi_{\mathrm{n}}$ and the workfunction $\phi_{o n}$ in flat band conditions. $V_{s p}-V_{s n}=$ Sum of $n$ and $p$ type barrier. SPV $n, S P V_{p}=n$ and $p$ type surface photovoltage.]

\begin{tabular}{|l|c|c|c|c|c|c|}
\cline { 2 - 7 } \multicolumn{1}{c|}{ GaAs (110) clivé } & $\begin{array}{c}\phi_{\mathrm{n}}-\phi_{\mathrm{on}} \\
(\mathrm{meV})\end{array}$ & $\begin{array}{c}\phi_{\mathrm{p}}-\phi_{\mathrm{on}} \\
(\mathrm{meV})\end{array}$ & $\begin{array}{c}\phi_{\mathrm{p}}-\phi_{\mathrm{n}} \\
(\mathrm{meV})\end{array}$ & $\begin{array}{c}\mathrm{V}_{\mathrm{p}}-\mathrm{V}_{\mathrm{v}} \\
(\mathrm{meV})\end{array}$ & $\begin{array}{c}\text { SPV } \\
(\mathrm{meV})\end{array}$ & $\begin{array}{c}\text { SPV } \\
(\mathrm{meV})\end{array}$ \\
\hline $\begin{array}{l}\text { Surface propre. } \\
\text { Défauts de clivage }\end{array}$ & 700 & -400 & 100 & 1100 & -350 & 70 \\
\hline Après recuit à $530^{\circ} \mathrm{C}$ & 350 & -550 & 300 & 1160 & -170 & 40 \\
\hline $2 \times 10^{4} \mathrm{~L}$ oxygène & 420 & -520 & 230 & 1130 & -330 & 70 \\
\hline Clivée $+1 / 4 \mathrm{mc} \mathrm{Ag}$ & 300 & -850 & 50 & 1170 & -240 & 30 \\
\hline
\end{tabular}

Tableau III. - Propriétés des surfaces clivées d'InP (110).

[(110) InP surface properties.]

\begin{tabular}{|l|r|r|r|r|c|c|}
\cline { 2 - 7 } \multicolumn{1}{c|}{} & $\begin{array}{r}\phi_{\mathrm{n}}-\phi_{\mathrm{on}} \\
(\mathrm{meV})\end{array}$ & $\begin{array}{c}\phi_{\mathrm{p}}-\phi_{\mathrm{op}} \\
(\mathrm{meV})\end{array}$ & $\begin{array}{c}\phi_{\mathrm{p}}-\phi_{\mathrm{n}} \\
(\mathrm{meV})\end{array}$ & $\begin{array}{c}\mathrm{V}_{\mathrm{p}}-\mathrm{V}_{\mathrm{w}} \\
(\mathrm{mV})\end{array}$ & $\begin{array}{c}\mathrm{SPV} \\
(\mathrm{mV})\end{array}$ & $\begin{array}{c}\mathrm{SPV} \\
(\mathrm{mV})\end{array}$ \\
\hline $\begin{array}{l}\text { Surface propre. } \\
\text { Défauts de clivage }\end{array}$ & 250 & -750 & $\leqslant 50$ & 100 & -120 & 700 \\
\hline $10^{3} \mathrm{~L}$ oxygène & 0 & 1000 & $\leqslant 50$ & 1000 & -50 & 550 \\
\hline 0,5 monocouche $\mathrm{Sb}$ & -150 & -1200 & $\leqslant 50$ & 1000 & -5 & 600 \\
\hline 0,5 monocouche $\mathrm{Ag}$ & 100 & -850 & $\leqslant 50$ & 950 & -80 & 280 \\
\hline 0,5 monocouche $\mathrm{Au}$ & 500 & -430 & $\leqslant 70$ & 930 & -70 & 230 \\
\hline 0,5 monocouche $\mathrm{Al}$ & -200 & 1150 & $\leqslant 50$ & 1000 & -5 & 500 \\
\hline 0,5 monocouche In & -120 & 1090 & $\leqslant 50$ & 1000 & -5 & 500 \\
\hline
\end{tabular}


Tableau IV. - Hauteurs de barrière et variation d'affinité électronique pour des surfaces clivées, exposées à l'oxygène et recouvertes d'Argent.

[Barrier height and electronic affinity variations for cleaved, annealed, oxygen exposed and Ag covered GaAs surfaces.]

\begin{tabular}{|l|c|c|c|}
\cline { 2 - 4 } \multicolumn{1}{c|}{ GaAs (110) } & $V_{\mathrm{sn}}(\mathrm{mV})$ & $V_{\mathrm{sp}}(\mathrm{mV})$ & $\Delta X(\mathrm{meV})$ \\
\hline Clivée propre. Défauts de clivage & -700 & 400 & $\leqslant 50$ \\
\hline Après recuit à $530^{\circ} \mathrm{C}$ & -600 & 300 & -250 \\
\hline $2 \times 10^{4} \mathrm{~L}$ oxygène & -640 & 330 & -220 \\
\hline Clivée $+0,5$ monocouche $\mathrm{Ag}$ & -810 & 340 & -510 \\
\hline
\end{tabular}

Tableau V. - Propriétés des surfaces clivées d'InP exposées à l'oxygène, recouvertes d'Ag, $\mathrm{Au}, \mathrm{Al}$, In.

[Cleaved InP surfaces: Barrier height and electronic affinity variations induced by oxygen exposure and $\mathrm{Sb}, \mathrm{Ag}, \mathrm{Au}, \mathrm{Al}$, In deposition.]

\begin{tabular}{|l|c|c|c|}
\cline { 2 - 4 } \multicolumn{1}{c|}{$\operatorname{InP}(110)$} & $V_{\mathrm{sn}}(\mathrm{mV})$ & $V_{\mathrm{sp}}(\mathrm{mV})$ & $\Delta X(\mathrm{meV})$ \\
\hline Clivée propre. Défauts de clivage & -250 & 750 & $\leqslant 50$ \\
\hline $10^{3} \mathrm{~L}$ oxygène & -180 & 820 & -180 \\
\hline $0,5 \mathrm{mc}-\mathrm{Sb}$ & -50 & 950 & -200 \\
\hline $0,5 \mathrm{mc}-\mathrm{Ag}$ & -280 & 650 & -200 \\
\hline $0,5 \mathrm{mc}-\mathrm{Au}$ & -250 & 650 & +240 \\
\hline $0,5 \mathrm{mc}-\mathrm{Al}$ & -50 & 920 & -230 \\
\hline $0,5 \mathrm{mc}-\mathrm{In}$ & -50 & 920 & -170 \\
\hline
\end{tabular}

Les positions du niveau de Fermi en surface $E_{\mathrm{Fs}}$ se déduisent directement de ces valeurs; elles impliquent la présence d'états donneur et accepteur, états dont nous avons discuté en détail les caractéristiques [15-20]. On a aussi déterminé des densités et positions en énergie qui sont en bon accord avec celles obtenues par d'autres auteurs utilisant d'autres techniques de caractérisation.

On peut donc conclure que sur les surfaces clivées propres, le photovoltage de surface peut être un moyen rapide de détermination de la barrière de surface et un support efficace pour la recherche de modèles d'états.

En fait, l'analyse simple précédente ne peut pas être appliquée à toutes les surfaces. En 
détermination de $\phi_{\text {on }}$ ou $\phi_{\text {op }}$ à partir des mesures Kelvin n'est pas possible ; les valeurs du photovoltage sur type $n$ et type $p$ fournissent seulement des indications sur l'importance de la barrière. C'est le cas des surfaces réelles.

3.2 SuRfaces AVEC ÉTATS EXTRINSĖQues. - On se place ici dans le cas de surfaces clivées dont les propriétés électroniques sont modifiées par recuit, interactions avec un gaz ou par dépôt métallique mince.

La figure 16 montre le suivi de $\phi$ et de SPV en fonction de la température du recuit et après dépôt d'argent sur surface clivée de GaAs. Les variations de $\phi$ et de SPV sur type $n$ et type $p$ sont corrélées et fournissent des informations sur la formation des barrières (variations corrélées des travaux de sortie et des photovoltages), sur l'apparition des dipôles de surface modifiant l'affinité électronique (fortes variations des travaux de sortie sans grande modification du photovoltage). Dans le cas du dépôt d'Ag et si seules intervenaient les modifications de barrière, les variations du photovoltage sur type $n$ et type $p$, devraient être accompagnées d'une évolution de $\phi$ de sens inverse à celle observée. Pour rendre compte des mesures, on doit conclure à une diminution de l'affinité électronique de plusieurs centaines de $\mathrm{meV}$. On aurait donc formation de défauts (lacunes anioniques ou cationiques) induisant des modifications de la barrière et d'affinité électronique).

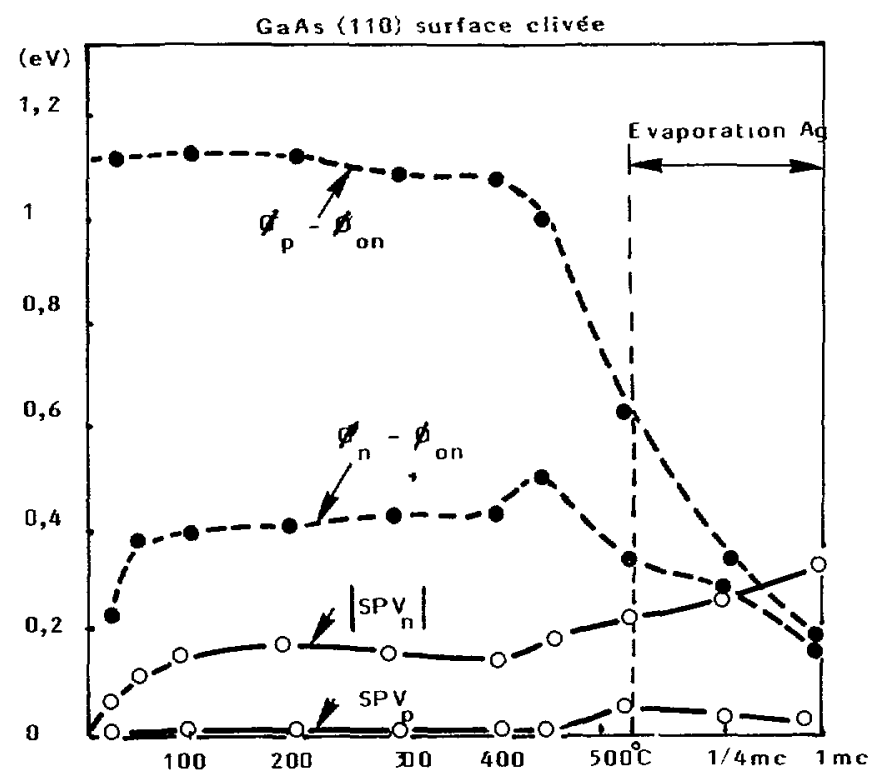

Fig. 16. - Evolution du travail de sortie et photovoltage de surface sur GaAs clivée en fonction de la température de recuit et après dépôt d'argent.

[GaAs workfunction and photovoltage variations versus the annealing temperature and after $\mathrm{Ag}$ deposit.]

Dans le cas des surfaces clivées (GaAs, InP et $\mathrm{GaSb}$ ) et exposées à l'oxygène, le suivi de $\phi$ et SPV en fonction de taux d'exposition (Figs. 17, 18, 19), nous a permis, comme dans le cas de la surface recuite, de déduire les barrières de surface $V_{s}$ et de suivre l'évolution du niveau de Fermi et de son ancrage en surface, en fonction de l'exposition à l'oxygène. La corrélation SPV $-\phi$ est encore étroite dans la gamme d'exposition $1 \mathrm{~L}-10^{2} \mathrm{~L}$ (Fig. 20) [la courbe SPV $\phi$ présente la même pente que la courbe relative à la surface clivée propre]. Au-delà de 


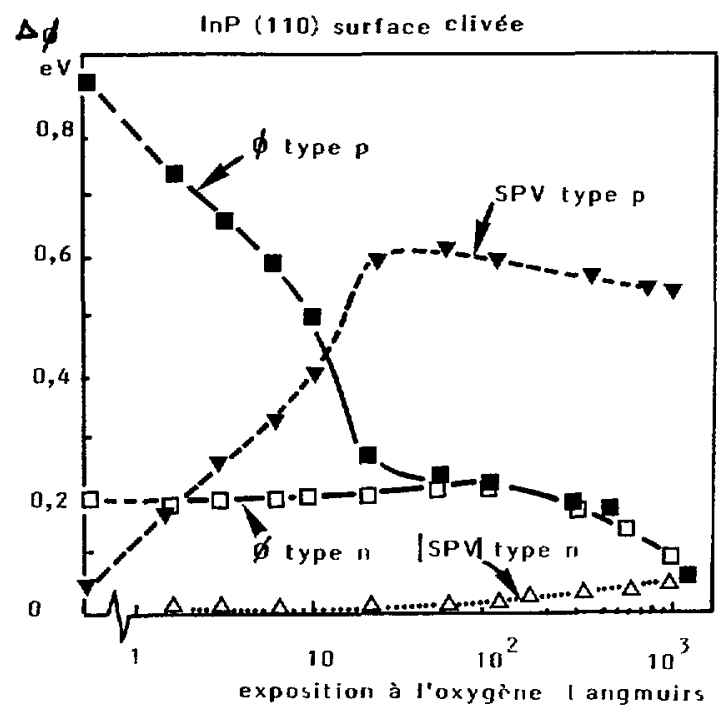

Fig. 17. - Evolution du travail de sortie et du photovoltage en fonction de l'exposition à l'oxygène. [Workfunction and photovoltage variations versus oxygen exposure.]

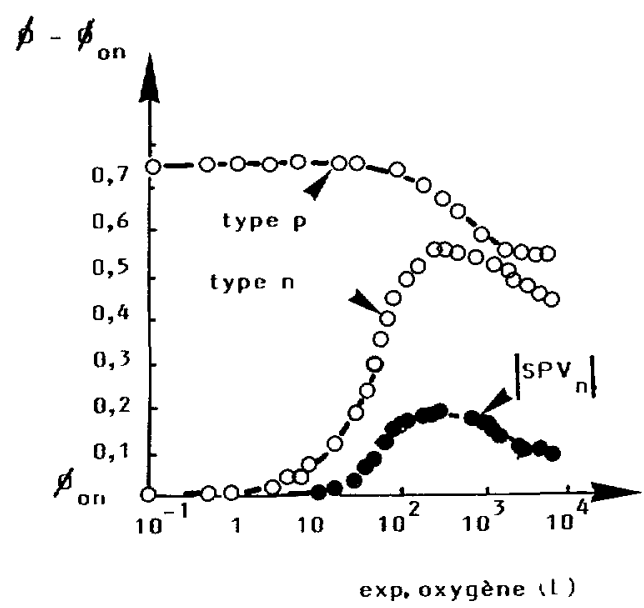

Fig. 18. - GaSb clivé : $n=10^{18} \mathrm{~cm}^{-3} p=7,6 \times 10^{16} \mathrm{~cm}^{-3}$ Evolution de $\phi$ et SPV en fonction de l'exposition à l'oxygène.

[n and $\mathrm{p}$ type $\mathrm{GaSb}$ workfunction and photovoltage variations versus oxygen exposure.]

cette exposition, une modification de l'affinité électronique de plusieurs centaines de meV doit être prise en compte, modification expliquée par un effet dipolaire.

L'effet de dépôt de fraction de monocouche de métal par exemple, sur les propriétés électroniques de surface peut être étudié de façon similaire en mesurant $\phi$ et SPV (Tab. II et III). Les résultats, dans ce cas aussi, mettent en évidence la création d'une barrière qui croît avec l'épaisseur du dépôt et sature lorsque la densité d'états créés est suffisante pour ancrer le niveau de Fermi. 


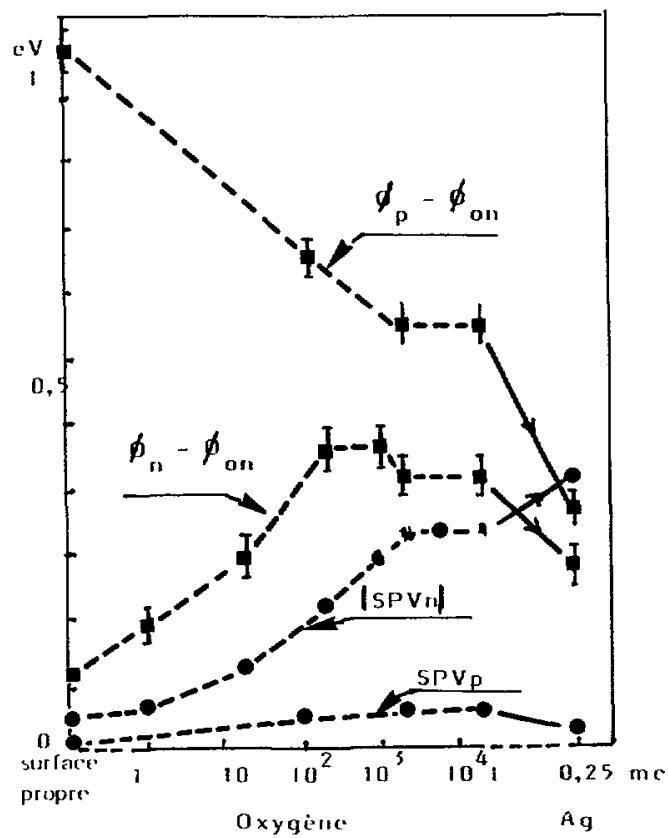

Fig. 19. - Evolution du travail de sortie et du photovoltage de surface de GaAs (110) de type n et $p$ après exposition à l'oxygène et après dépôt de 0,25 monocouche d'argent.

[ $\mathrm{n}$ and $\mathrm{p}$ type GaAs workfunction and photovoltage variations versus oxygen exposure and after 0.25 monolayer of Ag deposit.]

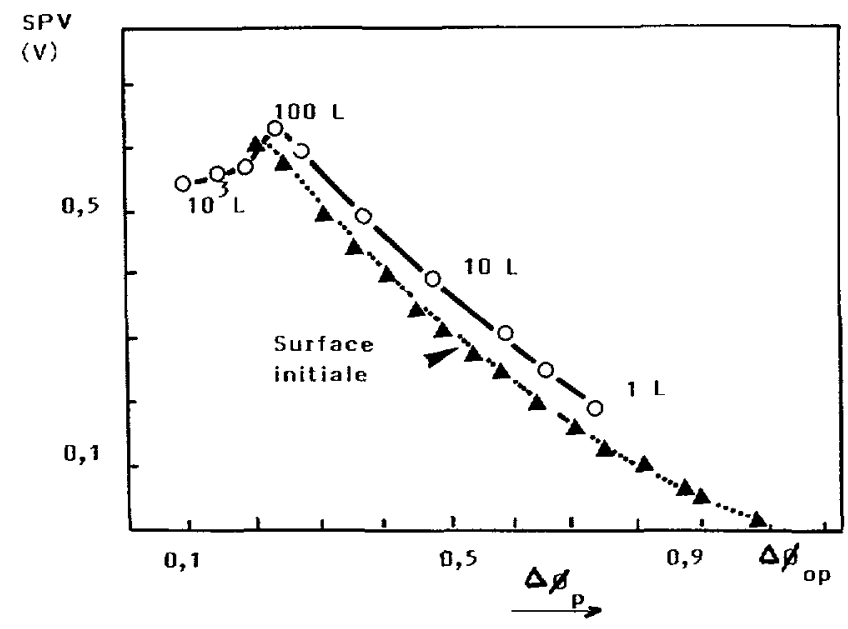

Fig. 20. - Variation du photovoltage en fonction du travail de sortie avant et après exposition à l'oxygène.

[Photovoltage variations versus workfunction before and after oxygen exposure.] 
L'étude de l'interaction et la détermination de $V_{\mathrm{sn}}$ et $V_{\mathrm{sp}}$, c'est-à-dire de $E_{\mathrm{Fsn}}$ et $E_{\mathrm{Fsp}}$ peut se faire en effectuant des mesures similaires sur échantillons de type p et de type $\mathrm{n}$. A partir des relations :

$$
\begin{aligned}
& q V_{\mathrm{sn}}=\phi_{\text {on }}-\phi_{\mathrm{n}}-\Delta \chi \\
& q V_{\mathrm{sp}}=\phi_{\mathrm{op}}-\phi_{\mathrm{p}}+\Delta \chi
\end{aligned}
$$

et des données des tableaux II et III et en supposant simplement qu'un même traitement conduit au même $\Delta \chi$ sur les échantillons de type $\mathrm{p}$ et de type $\mathrm{n}$, de qualité structurale équivalente, on s'affranchit de la connaissance de $\Delta \mathcal{X}$ et on a :

$$
q V_{\mathrm{sp}}-q V_{\mathrm{sn}}=\phi_{\mathrm{op}}-\phi_{\mathrm{on}}-\left(\phi_{\mathrm{p}}-\phi_{\mathrm{n}}\right) .
$$

La détermination de $V_{\mathrm{sn}}$ et $V_{\mathrm{sp}}$ implique l'évaluation de $\Delta \chi$. Or, $\Delta \chi$ n'est pas accessible directement par nos mesures car il n'est pas toujours possible de tracer des courbes significatives semblables à celles des figures $12,13,14,15$ qui devraient nous permettre d'obtenir les modifications des valeurs de $\phi_{\text {on }}$ et $\phi_{\text {op }}$.

Les valeurs de $V_{\text {sn }}, V_{\text {sp }}$ et $\Delta \chi$ pourraient cependant être calculées moyennant l'hypothèse qui semble expérimentalement acceptable en première approximation, que les courbes de variations de $S P V_{n}$ et $S P V_{p}$ en fonction de la barrière après modification de la surface se déduisent des courbes caractéristiques de la surface initiale par une homothétie de même rapport. Les courbes sont modifiées d'un facteur $\alpha$ qui dépendrait du traitement de la surface ou de la nature et de la quantité de l'adsorbat. Si donc pour la surface initiale on a :

$$
\mathrm{SPV}_{\mathrm{n}}=f^{0}\left(V_{\mathrm{sn}}\right) \text { et } \quad \mathrm{SPV}_{\mathrm{p}}=g^{0}\left(V_{\mathrm{sp}}\right)
$$

après modification de la surface (recuit, exposition à l'oxygène, dépôt du métal), nous avons :

ou

$$
\begin{gathered}
\mathrm{SPV}_{\mathrm{n}}=f\left(V_{\mathrm{sn}}\right) \text { et } \mathrm{SPV}_{\mathrm{p}}=g\left(V_{\mathrm{sp}}\right) \\
\frac{f\left(V_{\mathrm{sn}}\right)}{f^{0}\left(V_{\mathrm{sn}}\right)}=\frac{g\left(V_{\mathrm{sp}}\right)}{g^{0}\left(V_{\mathrm{sp}}\right)}=\alpha .
\end{gathered}
$$

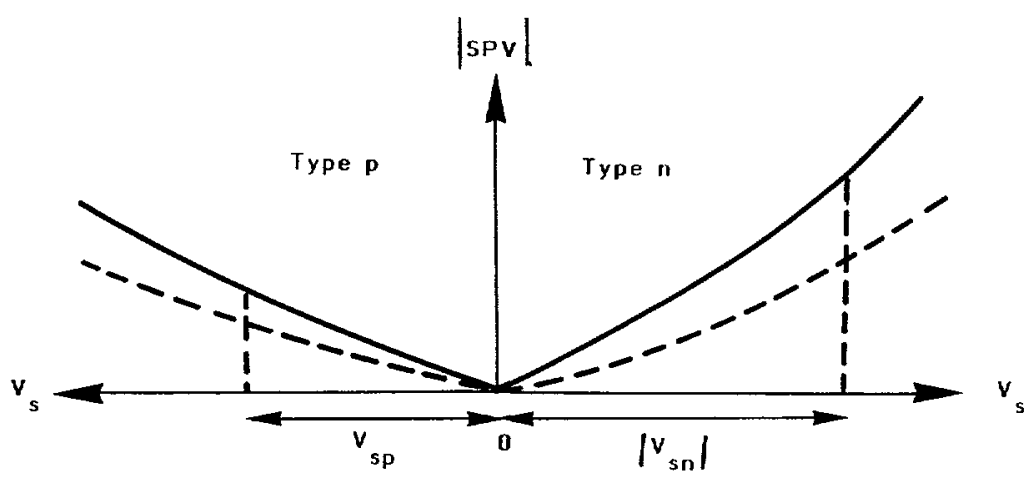

Fig. 21. - Courbes de variations du photovoltage en fonction de la barrière de surface avant et après modification de la surface $\frac{f\left(V_{\mathrm{sa}}\right)}{f^{0}\left(V_{\mathrm{sp}}\right)}=\frac{g\left(V_{\mathrm{sp}}\right)}{g^{0}\left(V_{\mathrm{sp}}\right)}=\alpha$.

[Photovoltage variations versus surface barrier height before and after the surface has been modified.] 
Les données des tableaux II et III fournissent les valeurs de $\left(V_{\mathrm{sp}}-V_{\mathrm{sn}}\right), \mathrm{SPV}_{\mathrm{n}}$, $\mathrm{SPV}_{\mathrm{p}}$ et le rapport $\frac{\mathrm{SPV}_{\mathrm{n}}}{\mathrm{SPV}}$ Les valeurs de $V_{\mathrm{sn}}$ et $V_{\mathrm{sp}}$ se déduisent alors graphiquement à partir des courbes du type figure 21 . Les valeurs de $\Delta \chi$ peuvent être ensuite directement calculées à l'aide des équations (9) et (10). Les valeurs ainsi obtenues portées tableaux IV et $V$ ne sont qu'indicatives en raison de l'approximation que nous avons faites sur le photovoltage. Elles constituent des ordres de grandeurs raisonnables et sont en bon accord avec les résultats publiés. Elles sont aussi en bon accord avec les résultats obtenus à partir de l'étude des diodes. En effet, lorsque la couche de métal déposée est suffisante pour ancrer le niveau de Fermi, la barrière déduite de nos expériences est la même que celle obtenue à partir des caractéristiques des diodes. On peut donc conclure que le $\Delta \boldsymbol{X}$ déduit de nos mesures est correct. Ce $\Delta \chi$, induit donc par dépôt, tend vers une valeur finale qui ajuste le travail de sortie du semiconducteur et le travail de sortie du métal.

\section{Conclusion.}

$\mathrm{Au}$ cours de cet article, nous nous sommes efforcés d'illustrer quelques possibilités de caractérisation des états de surfaces intrinsèques ou extrinsèques par la mesure du photovoltage SPV de surface.

Un rappel succinct des modèles de base du photovoltage, accompagné du tracé de quelques courbes caractéristiques des variations de barrière pour des échantillons de matériaux III-V nous a tout d'abord permis de préciser, pour des conditions d'expériences courantes, la contribution de différents paramètres au SPV. Nous avons établi numériquement l'importance de l'injection nécessaire à l'apparition du photovoltage, à l'applatissement de la barrière ; nous avons mis en évidence le rôle du dopage, des sections efficaces de capture des états... Les effets attendus dans quelques cas types étant ainsi précisés numériquement, nous avons appréhendé et interprété nos résultats expérimentaux dans des conditions plus satisfaisantes. Nous en avons déduit quelques conclusions qui se résument comme suit :

- Dans tous les cas, pour des intensités de flux de l'ordre de $5 \times 10^{17}$ photons. $\mathrm{cm}^{-2} \mathrm{~s}^{-1}$, le photovoltage indique le sens de la barrière $V_{s^{*}}$ S'il constitue une image de la barrière, il n'est cependant pas égal à $V_{\mathrm{s}}$. Dans les conditions expérimentales classiques, on ne peut pas espérer aplatir complètement les bandes.

- La réponse de la surface dépend de la nature du semiconducteur, des traitements, des perturbations de surface. La réponse photovoltaïque de surface clivée propre est celle qui se rapproche le plus du modèle théorique simple proposé. Ceci résulte très probablement du nombre réduit de paramètres et de la faible densité d'états de surface. Dans la bande interdite une perturbation importante de la surface par dépôt du métal (densité d'états élevée, défauts...) fait intervenir divers paramètres inconnus, qui rendent l'interprétation du SPV complexe. Cependant, les tendances mises en évidence par la théorie sont confirmées par les résultats expérimentaux. Une modélisation basée sur des modèles simplifiés permet d'interpréter les résultats et d'obtenir des caractéristiques raisonnables des états.

- Le photovoltage étudié sur type $n$ et $\mathrm{p}$ et associé aux études du travail de sortie peut fournir des informations sur l'ancrage et la position du niveau de Fermi en surface et donc permettre une caractérisation «préliminaire » des états de surface.

Malgré la complexité des mécanismes qui interviennent et qui font que son utilisation dans l'étude des propriétés de surface doit être prudente on doit conclure que le photovoltage peut être une source importante d'informations fiables sur les surfaces des composés III-V et sur leurs modifications sous l'effet de divers traitements et ce sans que doivent être prises en compte des perturbations des grandeurs à mesurer induites par la méthode comme c'est le cas en photoémission. 


\section{Bibliographie}

[1] Berz F., Surface Physics of phosphors and semiconductors, C. G. Scott et C. E. Reed Eds. (Academic Press, London, 1975).

[2] BRILlSON L. J., Thin Solid Films 89 (1982) 4.

[3] Ismail A., Palau J. M., Lassabatère L., Appl. Surf. Sci. 17 (1984).

[4] Brillson L. J., KRuger D. W., Surf. Sci. 102 (1981) 518.

[5] Dinan J. H., Galbraith L. K., Fisher T. E., Surf. Sci. 26 (1971) 587.

[6] Galbraith L. K., Fisher T. E., Surf. Sci. 30 (1972) 185.

[7] BRILLSON L. J., Surf. Sci., Rep. 2 n 2 (1982).

[8] Spicer W. E., Eglash S., Lindau I., Su C. Y. and Skeath P. R., Thin Solid Films 89 (1982) 447.

[9] Lagowski J., Balestra C. L., Gatos H. C., Surf. Sci. 29 (1972) 213.

[10] Mönch W., Clemens H. J., Górlich S., Enninghorst R., Gant H., J. Vac. Sci. Technol. 19 (1981) 525.

[11] Soonckindt L. (1978), Bonnet J. (1981), Palau J. M. (1982), Ismail A. (1984), Thèses, Université de Montpellier II, Sciences et Techniques du Languedoc, France.

[12] SIEFERT J. M., Thèse, Paris-Sud (Orsay) France (1986).

[13] Aldao C. M., Waddill G. D., Benning P. J., Capasso C., Weaver J. H., Phys. Rev. B 41 (1990) 6092.

[14] Waddill G. D., Tadahiro Koneda, Yang Y. N., Weaver J. H., Phys. Rev. B 41 (1990) 10283.

[15] Ismail A., Palau J. M., Lassabatère L., Rev. Phys. Appl. 19 (1984) 197.

[16] Ismail A., Ben Brahim A., Palau J. M., Lassabatere L., Surf. Sci. 162 (1985) 195.

[17] Ismail A., Ben Brahim A., Palau J. M., Lassabatère L., Surf. Sci. 164 (1985) 43.

[18] Ismail A., Palau J. M., Lassabatère L., J. Appl. Phys. 60 (1986) 1730.

[19] Ismail A., Ben Brahim A., Lassabatère L., Lindau I., J. Appl. Phys. 159 (1986) 485.

[20] Ismail A., Ben Brahim A., Palau J. M., Lassabatère L., Lindau I., J. Vac. Sci. Technol. 361 (1986) 217.

[21] Bonnet J., Soonckindt L., Ismail A., Lassabatère L., Thin Solid Films 151 (1987) 103.

[22] Palau J. M., Bonnet J., J. Phys. F., Sci. Instrum. 21 (1988) 674.

[23] Ismail A., Palau J. M., Lassabatère L., J. Phys. France 45 (1984) 1717. 